\title{
Dynamics of Antibiotic Prescription in Royal Hampshire County Hospital in November 2016: A Prospective Study
}

Kathrine Lee-A-Ping, ${ }^{1}$ Kordo Saeed, 2 Matthew Dryden, 3 Gavin Sim. ${ }^{4}$

\begin{abstract}
Background: Antimicrobial Stewardship and The Start Smart - Then Focus strategy provide guidelines aimed at improving the increasing trend of antibiotic resistance. The aim of this study was to assess whether antibiotics were being prescribed at Royal Hampshire County Hospital (a district general hospital), in accordance with the hospital's and the NICE guidelines and whether this followed the Start Smart - Then Focus approach. Methods: During November 2016, medical notes of 12 randomly selected in-patients of Royal Hampshire County Hospital on 45 antibiotics, were used to measure the dynamics of their prescriptions. Results: $91 \%$ of the 45 prescriptions were in accordance with hospital guidelines, $82 \%$ of cases had appropriate samples sent before commencing antibiotics, $5 \%$ out of $27 \%$ had a planned switch from intravenous administration to oral (the remaining $73 \%$ were initially started on oral regimes) and $80 \%$ had planned stop dates. Conclusion: Appropriate samples, stop dates, planning and documentation in patient notes must be improved with regards to antibiotic use.
\end{abstract}

Key Words: Anti-Bacterial Agents, Drug Resistance, Microbial (Source: MeSH-NLM).

\section{Introduction}

Antimicrobial resistance (AMR) is defined as the loss of effectiveness of antimicrobial agents and has become an emergent problem within the last few years. ${ }^{1}$ This resistance stems from overuse or rather incorrect use of antimicrobials. ${ }^{2}$ Microorganisms develop resistance when there is strong selection pressure from antibiotic use. (NICE Guidance). Overuse of antibiotics inappropriately is the major driver of resistance. 3 A recent BMJ Analysis piece was published which stated that an expert group refuted the age-old theory of the "antibiotic course", suggesting that there is no evidence that stopping a course of antibiotics early encourages resistance but rather taking antibiotics for longer than necessary is what drives resistance. 4 However, current general advice is to complete the course exactly as prescribed by the doctor. 4

Either way, patients with resistant infections have higher health care costs versus patients with non-resistant infections since resistant organisms generally result in longer hospital admissions, additional tests and the need for more expensive treatments. (World Health Organization). AMR not only leads to higher overall health care costs, but also increases risk of spread of infection to others as drugs become ineffective. 5

Antimicrobial Stewardship refers to coordinated interventions designed to improve and measure the appropriate use of antimicrobials by promoting the selection of the optimal antimicrobial drug regimen, dose, duration of therapy, and route of administration. It is becoming more crucial to improve the prescribing of antibiotics to help curb this increase in resistance by ensuring treatment standards are met and kept. ${ }^{6-7}$

Correct prescription of antibiotics not only curbs the rates of resistance; it can also be potentially lifesaving for patients. Studies have shown that effective administration of the correct antimicrobial agents within the first hours of a patient being diagnosed with sepsis or septic shock can lead to increased survival. One study showed that correct initiation of antimicrobial therapy in the first hour after a patient went into septic shock, showed an increased survival by $80 \%$ to hospital discharge. 7 In addition, each hour of delay in prescription over the ensuing six hours was associated with an average decrease in patient survival of $7.6 \% .8$ The concept of Start Smart Then Focus is geared towards this and other aspects of antibiotic stewardship and comprises various steps to follow when beginning any course of antibiotics.

Antimicrobial stewardship is a key part of the UK Five Year Antimicrobial Resistance Strategy and the 2011 Chief Medical officers (CMO) objections report.9-10 The core goals of stewardship are to reduce the emergence and rise of AMR by improving patient care. These goals are achieved by following the steps of the Start Smart Then Focus toolkit. ${ }^{11}$ The toolkit is meant to be enforced by strong clinical leadership. In any Trust, all members of the medical team should be in contact with a consultant microbiologist/ infectious disease specialist and the antimicrobial pharmacist. 8 The following extract from the Start Smart Then Focus Toolkit describes the steps.9, 11-12

\section{"Start Smart - this means:}

- do not start antimicrobial therapy unless there is clear evidence of infection

- $\quad$ take a thorough drug allergy history

- $\quad$ initiate prompt effective antibiotic treatment within one hour of diagnosis (or as soon as possible) in patients with severe sepsis or life-threatening infections. Avoid inappropriate use of broad-spectrum antibiotics

- comply with local antimicrobial prescribing guidance

- document clinical indication (and disease severity if appropriate), drug name, dose and route on drug chart and in clinical notes

- $\quad$ include review/stop date or duration

1 St. George's University, School of Medicine, Grenada, West Indies.

MB ChB MSc FRCPath, Consultant Microbiologist/ Hampshire Hospitals NHS Foundation Trust, Honorary Senior Lecturer/University of Southampton, Royal Hampshire County 2 Hospital, Romsey Road, Winchester, United Kingdom.

${ }_{3}$ MD FRCPath FRCPS (Glasgow), Consultant and Hon Senior Lecturer, Department of Microbiology and Infection, Hampshire Hospitals NHS Foundation Trust \& Rare and Imported Pathogens Department, PHE, Porton. University of Southampton and Professor, Dept of Medicine, St Georges University, Grenada.

4 MPharm, Pharmacist at Royal Hampshire County Hospital, Romsey Road, Winchester, United Kingdom. 
- obtain cultures prior to commencing therapy where possible (but do not delay therapy)

- $\quad$ prescribe single dose antibiotics for surgical prophylaxis where antibiotics have been shown to be effective

- document the exact indication on the drug chart (rather than stating long term prophylaxis) for clinical prophylaxis

Then Focus - this means:

- $\quad$ reviewing the clinical diagnosis and the continuing need for antibiotics at 48-72 hours and documenting a clear plan of action - the 'antimicrobial prescribing decision'

- the five 'antimicrobial prescribing decision' options are:

- 1. Stop antibiotics if there is no evidence of infection

- 2. Switch antibiotics from intravenous to oral

- 3. Change antibiotics - ideally to a narrower spectrum - or broader if required

- 4. Continue and document next review date or stop date

- 5. Outpatient Parenteral Antibiotic Therapy (OPAT)

- $\quad$ it is essential that the review and subsequent decision is clearly documented in the clinical notes and on the drug chart where possible example: stop antibiotic"8

The aims of this study were to assess the trend of how antibiotics are prescribed at Royal Hampshire County Hospital during the month of November 2016 and to compare whether antibiotics are being prescribed in the correct manner in accordance with the Start Smart Then Focus toolkit, the hospital's MicroGuide and the NICE guidelines. The hypothesis for this study was that there would possibly be a lack of correct prescription in accordance with national guidelines as human factors always play a role.

Our objective was to give an insight on some challenging aspects of antibiotic stewardship including the general trend of antibiotic use and compliance with the elements of Start Smart Then Focus. It was hoped that this study may serve as an educational tool to make improvements depending on the study's results. ${ }^{13}$ The results of this study can also be used to provide teaching points for staff to improve safer prescribing of antibiotics, help with curbing the growing problem of antibiotic resistance and ultimately improve patient care. ${ }^{14}$

\section{Methods}

This prospective study was completed as an audit in Royal Hampshire County Hospital during November 2016, in which twelve patients were selected on various wards within the hospital to assess how their antibiotics were prescribed based on the Start Smart Then Focus concept. The hospital's computerized system called JAC was used to generate a list of all inpatients currently on antibiotic medication, of which twelve were randomly across seven different adult wards. For the purpose of this study, only adult patients were chosen, and no paediatric patients were included. The sample size for the study was limited to 12 patients due to time constraints of the study's main conductor and time limit with the infection department. The twelve patients were found to be on a combination of 45 different antimicrobial agents which were used for the study. Once a patient was selected, their medications were reviewed with a specific focus on the antimicrobial agents that had been prescribed.

The hospital has around 300 beds and based on unpublished point prevalence audit data at each point in time, $30 \%$ of inpatients are on antibiotics i.e. the cohort in this audit represents $40 \%$ of the patients who had had antibiotics during the study period. Although this figure may not neatly represent the general population and prescribing practices of the hospital, it does give an insight into general trends and should identify any major issues.

The hospital's online App, MicroGuide, was used to determine whether the antimicrobial agents reviewed were prescribed according to the hospital's guideline. MicroGuide, is the local antibiotic guideline for HHFT provided through an online application (App). The App is owned by Horizon Strategic Partners Limited, was originally created to allow pharmacists to create, update and publish antimicrobial guidelines to their physicians' mobile devices to have the most up to date guidance available at the point of care. ${ }^{10}$ The company's aim has now evolved so that MicroGuide will facilitate better communications between clinicians and their Medical Organisations. MicroGuide's clinical content is constantly being updated and in 2015, a newer version of MicroGuide was released by Horizon that provided a greater number of guidelines. All of these guidelines were made available to clinicians by the use of a single mobile App creating a single point of access to the information. ${ }^{15}$

The name of the antimicrobial agent, the dose, frequency, route of administration and clinical indication for selection of that particula agent were of interest in this study. The study also focused to see if adequate documentation of all the above criteria were correctly made in the patient notes. Other focuses of the study included whether appropriate samples were taken before commencing antibiotics, if review dates were agreed on, if a stop date had been decided and if there were plans to switch to oral antibiotics when appropriate. If there was missing data from patient notes, this was documented in the study to show proof of lack of documentation.

This audit did receive approval by the hospital's Audit board. There was no need to acquire approval from a local Research Ethics board as patients did not have to sign an informed consent to take part since they were not receiving any interventional treatment. The patients' medications were being monitored and the study had no personal interaction with the patients themselves. There was no need for any statistical analysis to be run on the collected data as the information collected was to be used for retrospective audit purposes of monitoring antimicrobial prescription habits within the hospital and there was no potential source of bias.

\section{Results}

Based on the data collected, the twelve randomly selected patients (five females and seven males ranging from 28 to 92 years of age) who were from various wards, were found to be on an extensive list of 45 different combinations of antimicrobial agents over the course of their admission (Table 1).

However, indication for antimicrobial choice was not documented in the medical notes of one patient and two patients were missing dosage information of the agent they were on. The patients' initials, ages and the ward they were on were all documented in addition to the names of the drugs, the dosages, and routes of administration (Table 1). Additionally, for this cohort, $73 \%$ of cases did not require a switch from intravenous antibiotics to oral antibiotics as the patients were initially started on oral antibiotics as shown in Figure 1. However, of the

Figure 1. Planned Switch from Intravenous Route $\rightarrow$ Oral Route of 45 Antimicrobial Agents Prescribed to 12 Inpatients at Royal Hampshire County Hospital in November 2016.

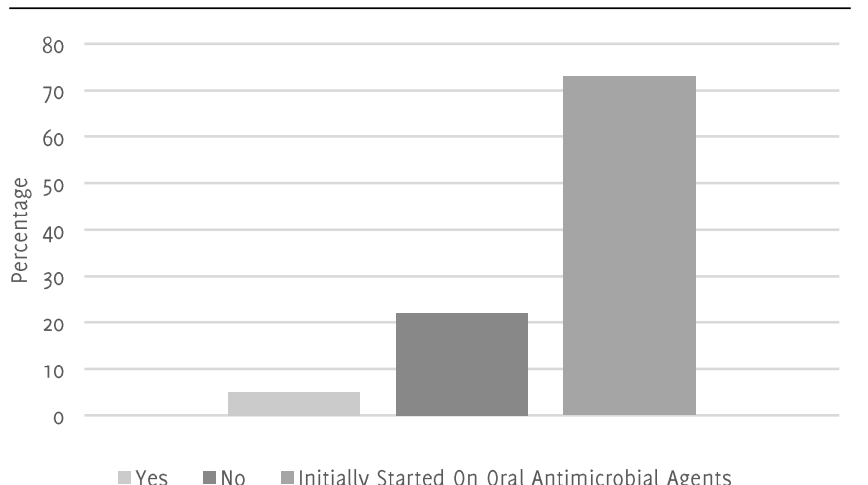


Table 1. Information Regarding 45 Antimicrobial Agents Prescribed to 12 Inpatients at Royal Hampshire County Hospital in November 2016.

\begin{tabular}{|c|c|c|c|c|c|c|}
\hline Patients & Sex & Age & Ward & Antimicrobial Agent Name & Dose & Route of Administration \\
\hline D.P & $\mathrm{M}$ & 59 & Clarke & $\begin{array}{l}\text { Co-amoxiclav } \\
\text { Co-amoxiclav } \\
\text { Metronidazole } \\
\text { Amoxicillin }\end{array}$ & $\begin{array}{c}1200 \mathrm{mg} \\
400 \mathrm{mg} \text { TDS } \\
1000 \mathrm{mg}\end{array}$ & $\begin{array}{l}\text { IV Bolus } 8 \text { hourly } \\
\text { Oral } \\
\text { Oral } \\
\text { Oral }\end{array}$ \\
\hline E.S & $\mathrm{F}$ & 86 & Shawford & $\begin{array}{c}\text { Piperacillin/Tazobactam } \\
\text { Co-trimoxazole } \\
\text { Co-trimoxazole }\end{array}$ & $\begin{array}{l}4.5 \text { g } 8 \text { hourly } \\
960 \mathrm{mg} \mathrm{BD} \\
960 \mathrm{mg}\end{array}$ & $\begin{array}{l}\text { IV infusion } \\
\text { Oral } \\
\text { IV infusion }\end{array}$ \\
\hline G.B & $\mathrm{F}$ & 73 & Victoria & $\begin{array}{l}\text { Co-trimoxazole } \\
\text { Clindamycin } \\
\text { Clarithromycin } \\
\text { Co-trimoxazole }\end{array}$ & $\begin{array}{c}960 \mathrm{mg} \mathrm{BD} \\
1200 \mathrm{mg} \\
500 \mathrm{mg} \\
960 \mathrm{mg}\end{array}$ & $\begin{array}{c}\text { Oral } \\
\text { IV infusion } \\
\text { Oral STAT } \\
\text { Oral STAT }\end{array}$ \\
\hline R. M & $\mathrm{M}$ & 69 & Victoria & $\begin{array}{l}\text { Co-amoxiclav } \\
\text { Clindamycin } \\
\text { Flucloxacillin } \\
\text { Flucloxacillin } \\
\text { Flucloxacillin } \\
\text { Flucloxacillin } \\
\text { Co-amoxiclav } \\
\text { Clindamycin }\end{array}$ & $\begin{array}{c}1200 \mathrm{mg} \text { TDS } \\
300 \mathrm{mg} \\
2 \mathrm{~g} \text { QDS } \\
1000 \mathrm{mg} \text { QDS } \\
2000 \mathrm{mg} \text { QDS } \\
2 \mathrm{~g} \text { QDS } \\
1200 \mathrm{mg} \\
300 \mathrm{mg} \text { QDS }\end{array}$ & $\begin{array}{c}\text { IV Bolus } \\
\text { Oral STAT } \\
\text { IV infusion } \\
\text { Oral } \\
\text { Oral } \\
\text { IV infusion } \\
\text { IV Bolus STAT } \\
\text { Oral }\end{array}$ \\
\hline $0 . C$ & $\mathrm{~F}$ & 92 & Freshfield & $\begin{array}{l}\text { Pipercillin/Tazobactam } \\
\text { Co-trimoxazole }\end{array}$ & $\begin{array}{c}4.5 \mathrm{~g} \\
960 \mathrm{mg} \mathrm{BD}\end{array}$ & $\begin{array}{l}\text { IV infusion STAT } \\
\text { IV infusion }\end{array}$ \\
\hline H. J & M & 91 & Kemp Welch & Co-amoxiclav & $500 \mathrm{mg}$ & Oral \\
\hline V. C & $\mathrm{F}$ & 31 & Bartlett & Ciprofloxacin & $500 \mathrm{mg}$ & Oral \\
\hline C. B & M & 28 & Kemp Welch & $\begin{array}{l}\text { Co-amoxiclav } \\
\text { Co-amoxiclav } \\
\text { Gentamicin }\end{array}$ & $320 \mathrm{mg}$ & $\begin{array}{c}\text { Oral } \\
\text { Oral STAT } \\
\text { IV infusion STAT }\end{array}$ \\
\hline A. C & $\mathrm{F}$ & 70 & Kemp Welch & $\begin{array}{c}\text { Fluconazole } \\
\text { Flucloxacillin } \\
\text { Piperacillin/Tazobactam } \\
\text { Metronidazole } \\
\text { Ciprofloxacin } \\
\text { Nystatin } \\
\text { Nystatin }\end{array}$ & $\begin{array}{c}50 \mathrm{mg} \\
7 \mathrm{~g} \\
4.5 \mathrm{~g} \\
500 \mathrm{mg} \\
500 \mathrm{mg} \\
4 \mathrm{ml} \\
4 \mathrm{ml}\end{array}$ & $\begin{array}{c}\text { Oral } \\
\text { IV infusion } \\
\text { IV infusion } \\
\text { IV infusion } \\
\text { Oral } \\
\text { Oral } \\
\text { Oral }\end{array}$ \\
\hline K. C & M & 55 & Shawford & $\begin{array}{l}\text { Ciprofloxacin } \\
\text { Co-trimoxazole }\end{array}$ & $\begin{array}{l}400 \mathrm{mg} \\
960 \mathrm{mg}\end{array}$ & $\begin{array}{l}\text { IV infusion } \\
\text { IV infusion STAT }\end{array}$ \\
\hline N. T & $M$ & 58 & McGill & $\begin{array}{l}\text { Flucloxacillin } \\
\text { Flucloxacillin } \\
\text { Doxycycline }\end{array}$ & $\begin{array}{c}2 \mathrm{~g} \\
2 \mathrm{~g} \\
100 \mathrm{mg}\end{array}$ & $\begin{array}{l}\text { IV infusion } \\
\text { IV infusion STAT } \\
\text { Oral }\end{array}$ \\
\hline T. J & M & 73 & Victoria & $\begin{array}{l}\text { Co-trimoxazole } \\
\text { Pipercillin/Tazobactam } \\
\text { Metronidazole } \\
\text { Rifaximin } \\
\text { Pipercillin/Tazobactam } \\
\text { Centamicin } \\
\text { Metronidazole }\end{array}$ & $\begin{array}{l}960 \mathrm{mg} \\
4.5 \mathrm{~g} \\
500 \mathrm{mg} \\
550 \mathrm{mg} \\
4.5 \mathrm{~g} \\
360 \mathrm{mg} \\
500 \mathrm{mg}\end{array}$ & $\begin{array}{c}\text { Oral } \\
\text { IV infusion } \\
\text { IV infusion } \\
\text { Oral } \\
\text { IV infusion STAT } \\
\text { IV infusion STAT } \\
\text { IV infusion STAT }\end{array}$ \\
\hline
\end{tabular}

Legend: BD - Twice a day, TDS -Three times a day, QDS - Four times a day.

remaining $27 \%$ of patients that were initially started on intravenous antibiotics, only $5 \%$ of cases had documented a plan to change the route of administration from intravenous to oral whereas $22 \%$ of cases had no plan or documented plan to change from intravenous to oral. Data regarding timeliness of antibiotics could not be gathered due to lack of documentation.

Unfortunately, this information was not initially recorded in the patients' notes and there is no way of gathering these details at this point in time. Figure 2 shows that $91 \%$ of antibiotic prescriptions were in accordance with hospital guidelines, $82 \%$ of cases had appropriate samples taken before commencing antibiotics, $80 \%$ had planned stop dates for antibiotic course and $5 \%$ of cases had a documented plan to switch the route of administration from intravenous to oral.

\section{Discussion}

According to the results of this small cohort, there is already a good result of $90 \%$ cases of prescribed antibiotics being compliant with the existing hospital guidelines which are for empiric prescribing before cultures are available and this is a key element of the Start Smart Then Focus protocol. However, in only $82 \%$ of the time, appropriate samples were taken and sent before starting antibiotics, which may be as a result of there being nothing to sample. Samples and cultures are important for identifying a specific organism or agent that is 
Figure 2: Dynamics of 45 Antimicrobial Agents Prescribed to 12 Inpatients at Royal Hampshire County Hospital in November 2016.

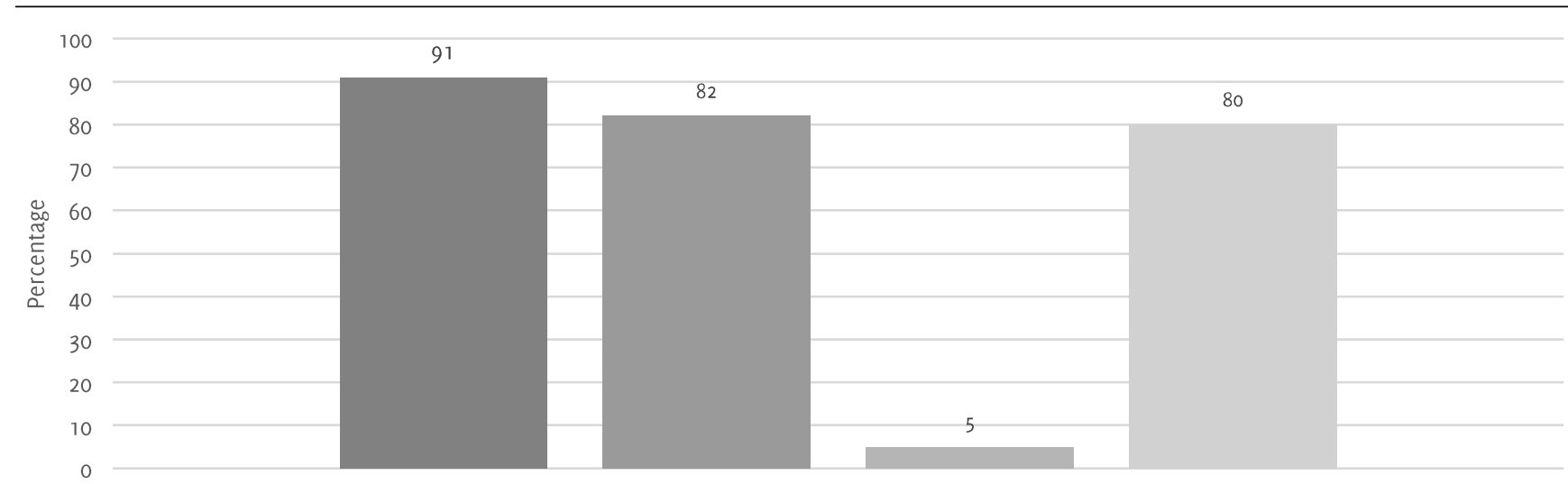

- Compliant with Guidelines $\quad$ Appropriate Samples Taken $\quad$ Planned Switch of Antimicrboial Agents From Intravenous Route $\rightarrow$ Oral Route $\quad$ Planned Stop Date

responsible for causing disease in the patient and targeted therapy or rationalization (the focus aspect of the process).

This is not only crucial for patient care, but also helps reduce the incidence of antimicrobial resistance which is a growing concern that has generally risen due to over prescribing and misuse of antibiotics. 5 Out of $27 \%$ of cases needing a switch from intravenous to oral there is only $5 \%$ having this plan documented, this is also a crucial aspect of patient safety. According to the English surveillance programme for antimicrobial utilisation and resistance (ESPAUR) report in 2015, there was a rise in bloodstream infections of Escherichia coli and Klebsiella pneumoniae due to increases in the number of people with antibiotic resistant infections. 5

Intravenous antibiotics should only be reserved for patients who require it as it leads to prolonged hospital stay and increases the chance for healthcare associated infections including intravenous line infections. If patients are well enough, there is no need to keep them on intravenous antibiotics and therefore no need for them to remain within hospital which can help reduce the burden of hospital resources and staff. Patients tend to do better when back in their homes, out of hospital, in an environment that is more comfortable for them. With this being the case, it is better to have patients switched to oral form antibiotics to help in the recovery process. ${ }^{11}$ Lastly, only $80 \%$ of cases had a documented stop date. This lack of a planned stop date along with other elements that we could not measure such as timeliness of prescription due to poor documentation require further education and increased awareness among prescribers. A potential stop date should always be agreed upon and documented correctly in-patient notes to aid in handing over and aid anyone reading the patient's notes. Timelines of antibiotics in septic patients are crucial to prevent excess mortality. According to Kumar et al. survival was $82.7 \%$ if effective antimicrobials were administered within 30 minutes of initial evidence of hypotension. ${ }^{8}$

Without documentation, it is not possible to comment on these practices which are again a crucial element of patient safety and improving survival from sepsis. Further training and education are required to improve these practices for all prescribers followed by a re-audit to assess the impact of this intervention for review and planned switches from intravenous to oral when prescribing antibiotics.

The study has a number of limitations including sample size of only 12 patients. However, 45 antimicrobial agents were prescribed in these patients, highlighting the complexity of some clinical situations and the challenges that doctors face in treating some cases with broad and combination of antibiotics.
Other limitations include the lack of ability to determine timeliness of antibiotics from making a diagnosis to write up of antibiotic prescription (in minutes) and to see the time between write up of antibiotic prescription to the time the dosage was given. Due to a lack of documentation in the patient notes about a clear diagnosis being established and lack of time recorded, it was difficult to assess this and include in the study. Similarly, there was lack of documentation of the time of write up of prescription again making this measure unable to be assessed for the study. These will be used as feedback points for the prescribers in the hospital.

The results of this study can be used as a generalization to highlight the short comings of prescription practices and can serve as a model for improvement. All health care professionals should focus on all steps of the Start Smart Then Focus approach with regards to antibiotic use and follow the guidelines. Doctors should consult Microbiology/ infection specialist before prescribing a patient antibiotic medication when the clinical picture is challenging, or previous microbiology necessitate this. All health care workers should provide accurate and correctly documented information in medical notes including time and date as well as have a planned stop date.

\section{Conclusion}

Based on the results of this study, in November 2016, at Royal Hampshire County Hospital $91 \%$ of prescriptions were in accordance with hospital guidelines, $82 \%$ of cases had appropriate samples sent before commencing antibiotics, $5 \%$ out of $27 \%$ had a planned switch from intravenous administration to oral and $80 \%$ had planned stop dates. This shows that there is a need for improvement with regards to appropriate samples needing to be taken before commencing antimicrobial therapy. Proper planning of stepping down treatment by switching from intravenous to oral should also be made in addition to having clear stop dates agreed upon.

All this information should also be clearly documented in all patient notes to improve patient care and increase antimicrobial stewardship. Following the guidelines set out by the Start Smart - Then Focus program will greatly reduce the incidence of inappropriate antimicrobial use and therefore curb the rising rate of antimicrobial resistance. ${ }^{16-17}$ 


\section{References}

1. Costelloe C, Metcalfe C, Lovering A, Mant D, D Hay A. Effect of antibiotic prescribing in primary care on antimicrobial resistance in individual patients: systematic review and meta-analysis. British Medical Journal. 2010;304:c2096. doi:10.1136/bmj.c2096

2. Pathiraja. Antimicrobial Stewardship Start Smart - Then Focus: Guidance for Antimicrobial Stewardship for SHSCT [Internet]. 2015 Oct. Available from: http://www.southernguidelines.hscni.net/?wpfb_dl=220 [Accessed 22nd January 2017].

3. NICE Guidance. Antimicrobial Stewardship: Prescribing Antibiotics (KTT9). 2015 Jan. Available from: https://www.nice.org.uk/guidance/kttg/resources/antimicrobial-stewardshipprescribing-antibiotics-1632178559941 [Accessed 22nd January 2017].

4. Llewelyn M. J, Fitzatrick J. M, Darwin E, Tonkin-Crine S, Corton C, Paul J, et al. The antibiotic course has had its day. BMJ. 2017 Jul 26;358:j3418.

5. World Health Organization (WHO). World Health Organization Antimicrobial Resistance Fact Sheet Updated October 2017. http://www.who.int/mediacentre/factsheets/fs194/en/ [Accessed November 6th 2017].

6. NICE Guidance. Antimicrobial Stewardship: Systems and Processes for Effective Antimicrobial Medicine Use (NG15). 2015 Aug. Available from: https://www.nice.org.uk/guidance/ng15/resources/antimicrobial-stewardshipsystems-and-processes-for-effective-antimicrobial-medicine-use-1837273110469 [Accessed January 22nd 2017]

7. NICE Guidance. Antimicrobial Stewardship (QS121). 2016 Apr. Available from: https://www.nice.org.uk/guidance/as121/resources/antimicrobial-stewardship25545353537477 [Accessed 22nd January 2017]

8. Kumar A, Roberts D, E. Wood KE, Light B, Parrillo JE, Sharma S, et al. Duration of hypotension before initiation of effective antimicrobial therapy is the critical determinant of survival in human septic shock. Crit Care Med. 2006 Jun;34(6):1589-96.

9. Public Health England. Start Smart - Then Focus Antimicrobial Stewardship Toolkit for English Hospitals Updated March 2015 [Internet]. 2015 March. Available

from: https:/www.gov.uk/government/uploads/system/uploads/attachment_data/file /417032/Start_Smart_Then_Focus_FINAL.PDF [Accessed 24th January 2017].
10. Davies S. Annual Report of the Chief Medical Officer 2011: Volume Two. Infections and the Rise of Antimicrobial Resistance. Available from https://www.gov.uk/government/uploads/system/uploads/attachment_data/file 138331/CMO_Annual_Report_Volume_222011.pdf [Accessed 4th August 2017].

11. Start Smart Then Focus Prescribers checklist. Available from: https://www.gov.uk/government/uploads/system/uploads/attachment_data/file \$366944/Secondary_care_prescribers_checklist.pdf [Accessed 28th January 2017].

12. lechyd Cyhoeddus Cymru Public Health Wales. Antimicrobial Stewardship: "Start Smart - Then Focus" Guidance for Antimicrobial Stewardship for Hospitals in Wales. Available from: http://www.wales.nhs.uk/sitesplus/documents/888/Public\%20Health\%20Wales \%20Antimicrobial\%20Stewardship\%20Guidance.pdf [Accessed 28th January 2017].

13. Lockwood AR, Bolton NS, Winton MD, Carter JT. Formalization of an antimicrobial stewardship program in a small community hospital. Am J Health Syst Pharm. 2017 Sep 1;74(17 Supplement 3):S52-S60.

14. Barlam TF, Cosrove SE, Abbo LM, MacDougall C, Schuetz AN, Septimus EJ, et al. Implementing an antibiotic stewardship program; guidelines by the Infectious Diseases Society of America and the Society for Healthcare Epidemiology of America. Clin Infect Dis. 2016 May 15;62(10): 551-77.

15. MicroGuide. Available from: http://www.microguide.eu/ [Accessed 25th June 2017].

16. Huttner A, Harbarth S, Carlet J, Cosgrove S, Coossens H, Holmes A, et al. Antimicrobial Resistance: a global view from the 2013 World HealthcareAssociated Infections Forum. Antimicrob Resist Infect Control. 2013 Nov 18;2:31.

17. Berrevoets MA, Ten Oever J, Sprong T, van Hest RM, Groothuis I, van Heijl I, et al. Monitoring, documenting and reporting the quality of antibiotic use in the Netherlands: a pilot study to establish a national antimicrobial stewardship registry. BMC Infect Dis. 2017 Aug 15;17(1):565.

\section{Acknowledgments}

None.

Conflict of Interest Statement a Funding

The Authors have no funding, financial relationships or conflicts of interest to disclose.

\section{Author Contributions}

Conception and design the work/idea: KLAP, KS. Collect data/obtaining results: KLAP. Analysis and interpretation of data: KLAP, KS, GS. Write the manuscript: KLAP. Critical revision of the manuscript, Approval of the final version: KLAP, KS, MD, CS.

Cite as:

Lee-A-Ping K, Saeed K, Dryden M, Sim G. Dynamics of Antibiotic Prescription in Royal Hampshire County Hospital in November 2016: A Prospective Study. Int J Med Students. 2017;5(3):99-103. 